\title{
«Wir kranken am Schubladisieren»
}

\section{Daniel Lüthi}

Freier Journalist und Fotograf, Medientrainer, Bern

Zuerst einmal müssen Gemüse und Früchte an den richtigen Ort. "Tschabi», wie er in der Familie und von seinen Freunden genannt wird, war auf dem Märit, dem Berner Samstagsmarkt. Jetzt kommt in aufgewärmten kleinen Tassen ein feiner Espresso auf den Tisch. «Ja», sagt er in diesem entspannten Moment auf der sonnigen Terrasse seines Reiheneinfamilienhauses, «ich geniesse das Leben.»

\section{Dr. Rieux und die Pest}

Bald schon macht er klar, dass er damit das ganze Leben meint, also «Leben A» und «Leben B», Freizeit und Arbeit. «Die Idee einer Work-Life-Balance ist für mich eines der fremdartigsten Konzepte überhaupt», stellt er fest. «Das tönt ja so, als würde die Arbeit nicht zum Leben gehören. Für mich gibt es nur eine Life-Balance». Dieser gesamtheitliche Ansatz wird die ganze Begegnung prägen. Hansjakob Furrer lässt sich kaum themenweise durch ein Gespräch führen. Er spricht in langen Sequenzen und kommt vom einen zum andern - nicht weil er in seinen Gedanken sprunghaft wäre, sondern weil für ihn alles irgendwie zusammengehört oder irgendwo ineinander übergeht. «Wir kranken am Schubladisieren", sagt er, und formuliert damit sein prägendes Motto, das sich in allen Themenbereichen wiederfindet.

Wenn er zum Beispiel an seine Jugend denkt, kommt ihm sein Vater in den Sinn. «Er war Bauer. Sonntags nach dem Stall, und das erwartet man von einem Bau-

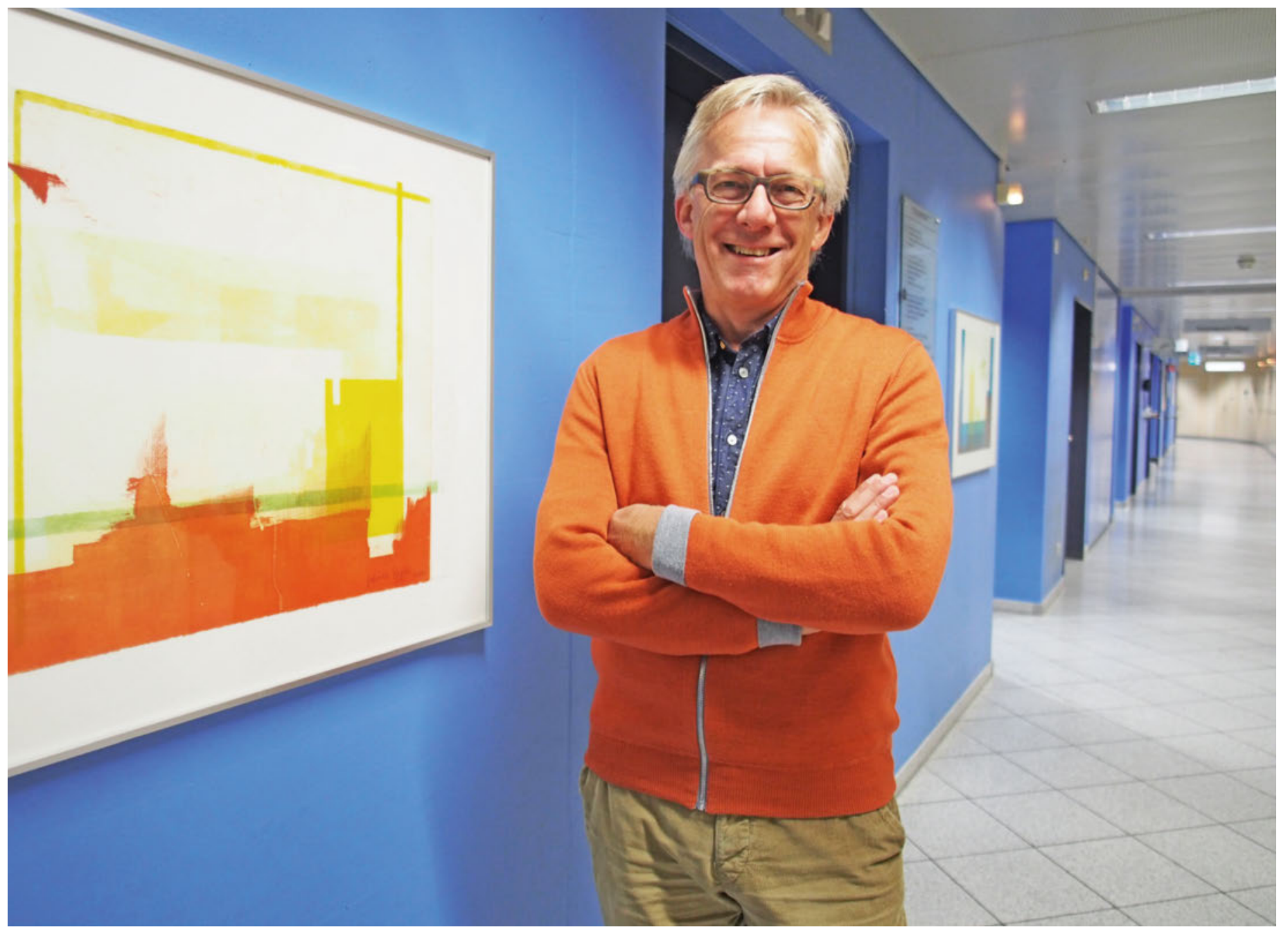


ern nicht, setzte er sich ans Klavier und spielte Bach.» Und wenn er sich an seinen Werdegang als Mediziner erinnert, macht er einen Ausflug in die Literatur. Er spricht von einer Figur aus dem Roman La peste von Albert Camus: Dr. Bernard Rieux, der in der algerischen Stadt Oran in den 1940er-Jahren Pest-Kranke pflegte. «Er sah die Leute sterben und hatte keine Mittel, sie zu heilen. Aber er war da, und das hatte einen Sinn. In der ganzen Absurdität des Sisyphos hatte er eine Aufgabe. Das faszinierte mich schon als Gymnasiast. Ich wollte auch etwas Sinnvolles tun für die Menschen, deshalb wurde ich Arzt.» Dazu eine Ergänzung im Sinn des erwähnten Mottos: «Ich stand immer mehr auf Camus als auf Sartre. Das Absolutistische war mir stets fremd, und bei Camus spielt stärker das Menschliche eine Rolle.»

\section{Ein Leben mit HIV}

Hausarzt war der Plan, dann aber verschlug es Hansjakob Furrer 1989 als Assistenzarzt in die neugeschaffene HIVSprechstunde des Berner Inselspitals. Er blieb sowohl dem Thema als auch der Institution bis heute treu.

«Damals, in der Anfangszeit, kam mir ganz stark wieder Dr. Bernard Rieux aus La peste in den Sinn. Ich realisierte: Ich war genau dort, wo er gewesen war. Die Entwicklungen rund um HIV und Aids waren prägend für mich», sagt Furrer. Deshalb ist für ihn jedes Jahr auch der Welt-Aids-Tag vom 1. Dezember ein wichtiges Ereignis. «Damals hatten viele das Gefühl, HIV gehe sie nichts an. Es war eine grosse Arbeit, in der Gesellschaft das Bewusstsein zu verankern, dass HIV eine grosse

\section{«Wo beginnt ein Bluthochdruck?»}

Gefahr für die ganze Gesellschaft und die ganze Welt ist.» Wichtig sei nicht nur die Öffentlichkeitsarbeit gewesen, sondern auch ein Umdenken im Gesundheitswesen. "Es ging darum, das Schubladendenken zu überwinden und zusammenzuarbeiten.» Neu seien für Kliniker und Labormediziner damals vor allem die häufigen opportunistischen Erkrankungen gewesen. «Die Abwehrstörung bei HIV war eine ganz andere als bei Transplantationen oder Krebstherapien.»

Die neue Situation interessierte nicht nur den Arzt, sondern auch den Naturwissenschaftler Furrer. "In einer gesamtschweizerischen Kohortenstudie sammelten - und sammeln - wir alle relevanten Daten in einer gemeinsamen Datenbank. So haben wir sogar neue Erreger entdecken können, mit denen unser Abwehrsystem im Normalfall gut umgehen kann, die bei einer Immunschwäche jedoch zum grossen Problem werden.»

\section{Hansjakob Furrer}

Prof. Dr. med. Hansjakob Furrer wurde 1959 als Bauernsohn in Henggart im Zürcher Weinland geboren. Er studierte Medizin an der Universität Zürich, wo er 1985 das Staatsexamen machte. 1989 kam er als Assistenzarzt ans Berner Inselspital, dem er bis heute treu geblieben ist. 1995 erhielt er seinen Facharzttitel in Innerer Medizin, im Jahr 2000

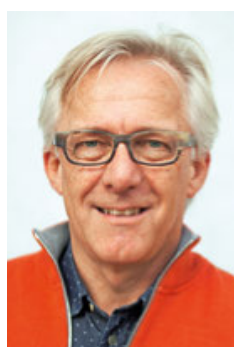
denjenigen für Infektiologie. Seit 2005 ist er Professor an der Universität Bern. Hansjakob Furrer ist verheiratet, Vater von drei erwachsenen Kindern und zweifacher Grossvater. Er lebt mit seiner Frau in Bern.

Ursachenforschung war das eine, die Entwicklung einer wirkungsvollen medikamentösen Therapie das andere. «Es ging 13 Jahre, bis wir eine vernünftige Kombinationstherapie entwickelt hatten.» Und heute? «Heute behandeln wir jede HIV-Infektion möglichst früh nach der Diagnose, unabhängig von der jeweiligen Abwehrschwäche. Neben dem Gesundheitsvorteil für den Patienten erreichen wir damit im Sinne von treatment as prevention auch, dass die HIV-Infektion nicht weitergegeben wird, denn gut behandelte HIV sind nicht mehr infektiös. Und: Wenn ein HIV-Patient eine Behandlung erträgt und bereit ist, die Medikamente wie vorgesehen einzunehmen, ist seine Lebenserwartung praktisch normal», bilanziert Furrer.

\section{Was ist normal?}

A propos «normal»: «Wo beginnt ein Bluthochdruck?», fragt Hansjakob Furrer fast ein wenig schelmisch. «Nach Lehrbuch bei 140 mm Hg. Und 139 ist normal?» Natürlich ist die Frage rhetorisch und die Antwort lautet «nein». "Solche Schubladen sind künstliche Konstrukte, sage ich meinen Studenten; biologische Phänomene sind meist kontinuierliche Entwicklungen, die ineinander übergehen, und es ist absurd zu denken, dass sich mit einem Millimeter Quecksilbersäule Unterschied im Blutdruck eine ganze Prognose schlagartig verändert.»

Insgesamt hat sich die Situation rund um HIV und Aids in unseren Breitengraden verändert bis «normalisiert», wobei genau dies wieder problematisch werden kann. «Die durchschnittliche heterosexuelle Bevölkerung hierzulande ist kaum mehr betroffen - mit Ausnahme von Leuten, die sich im Ausland anstecken. Bei einem Teil der Männer, die Sex mit Männern haben, ist ein Hochrisiko-Verhalten jedoch etabliert. Hier gibt es immer auch viele Neuinfektionen.» Wohl auch, weil das entsprechende Bewusstsein fehlt: «Kaum ein jun- 
ger Schwuler kennt heutzutage noch jemanden, der an Aids erkrankt oder gestorben ist.» HIV sei weitgehend therapierbar und bei uns unsichtbar geworden, sagt Furrer, "damit haben Sorglosigkeit und Gleichgültigkeit zugenommen. Auch deshalb beobachten wir, dass Geschlechtskrankheiten wie Chlamydien, Tripper oder Syphilis zunehmen.»

\section{Die neuen Bedrohungen}

Es gibt Verschiebungen in der Infektiologie, so auch bei Hansjakob Furrer. Als besonders bedrohlich erlebt auch er die zunehmenden Antibiotikaresistenzen. «Die Schweiz ist in dieser Hinsicht ebenfalls - vorläufig noch - eine Insel. Aber wenn ich mit Kollegen beispielsweise in Italien oder Griechenland spreche, erhalte ich den Eindruck, dass wir schon fast wieder so weit sind, wie in der Zeit vor den Antibiotika. Immer mehr Menschen sind besiedelt mit hochresistenten Keimen. Wenn diese Bakterien zu Infektionen führen, hat die Medizin nichts mehr anzubieten, was nützt.» Angesprochen auf seine engste Umgebung sagt er: «Ja, wir haben hier deswegen auch schon Todesfälle erlebt.»

\section{«Immer mehr Menschen sind besiedelt mit hochresistenten Keimen.»}

Die UNO ist alarmiert und die Schweiz auch. Auf Bundesebene ist die «Strategie Antibiotikaresistenzen (StAR)» lanciert worden. Hier arbeitet Hansjakob Furrer mit, und hier erlebt er einmal mehr, wie wichtig es ist, mit dem Schubladendenken aufzuhören. «Tierzucht, Landwirtschaft und Medizin müssen das Problem gemeinsam angehen. In der Diskussion zwischen Human- und Veterinärmedizinern zeigt sich erneut, wie alles miteinander verbunden ist. So kann ein Tierbakterium im menschlichen Darm sein Resistenz-Gen durchaus auf ein menschliches Bakterium übertragen.»

Schuld am grossen Problem sei auch die Medizin selbst. Zu lange habe sie Antibiotika zu extensiv und zu wenig fokussiert eingesetzt, sagt Furrer. «Früher warst du ein schlechter Arzt, wenn du eine Erkältung nicht mit Antibiotika behandelt hast. Und selber habe ich beispielsweise gelernt, dass du bei einer Lungenentzündung zwei bis drei Wochen lang Antibiotika geben musst. Heute wissen wir, dass bei einem günstigen Verlauf drei bis fünf Tage reichen können.» Ein Umdenken habe bei den Patienten und in der Ärzteschaft eingesetzt, «aber wir müssen Verläufe noch genauer beobachten, müssen noch besser abwägen, wo wir wie lange zuwarten und auf das körpereigene Immunsystem vertrauen können.»
Das Insel-Denken habe auch hier ausgedient: «Viele Leute, zum Beispiel Indien-Reisende, kommen mit hochresistenten Bakterien im Darm zurück.» Das sei zwar nicht eine unmittelbare Gefahr, «aber wenn wir bei einer Infektion später einmal Antibiotika einsetzen, manifestiert sich das Problem, denn dann werden die nicht-resistenten Bakterien getötet und die resistenten nehmen Überhand. Finden diese dann während einer Hospitalisation beispielsweise via einen Urindauerkatheter den Weg in die Harnblase und die Niere, können die Patienten an nicht behandelbaren Nierenbeckenentzündungen erkranken und sterben.»

\section{Hausgemachte Infektionen}

Womit wir bei einem weiteren Schwerpunkt von Hansjakob Furrer angelangt sind, der mit den Antibiotika-Resistenzen eng zusammenhängt: Seit er offiziell Direktor seiner Klinik ist, kann er gegenüber der Spitalleitung effektiver für die Spitalhygiene kämpfen. «In diesem Bereich können und müssen wir besser werden. Wir müssen unsere Überwachungs- und Reaktionssysteme ausbauen. Dafür brauchen wir Aus- und Weiterbildung, also mehr Personal und Geld. Der Stand der Spitalhygiene ist heutzutage eines der wichtigsten Qualitätsmerkmale eines Spitals», ist Furrer überzeugt. Und zunehmend auch ökonomischer Faktor: «Eine Spitalinfektion verteuert einen Fall um bis zu 15000 Franken. Bei hochresistenten Keimen verdoppeln sich die Kosten. Nosokomiale Infekte kosten allein das Inselspital Dutzende Millionen Franken pro Jahr.»

Die Glocken der etwas bedrohlich wirkenden Kirche nebenan signalisieren lautstark, wie schnell die Zeit vergangen ist. Ein wichtiges Thema im Leben von Hansjakob Furrer muss unbedingt noch angesprochen sein: Die Musik. Er singt im Chor der Kirche, die eben zum Aufbruch gemahnt hat, und er spielt möglichst regelmässig Saxophon, am liebsten Jazz und Blues. Früher tat er dies in einer Tanzband namens "Afrodisia». Jetzt tritt er ab und zu mit einem Metallurgen auf, der Gitarre spielt. Das Duo nennt sich «Plan B». Hansjakob Furrer begleitet seinen vielsagenden Kommentar dazu mit einem Schmunzeln: «Ich hatte nie einen Plan B. Jetzt schon.»

\section{Bildnachweis} Fotos Daniel Lüthi 